\title{
Properties of Recycled-Polyethylene Terephthalate/Polycarbonate Blend Fabricated by Vented Barrel Injection Molding
}

\author{
Wiranphat Thodsaratpreeyakul ${ }^{1}$, Putinun Uawongsuwan ${ }^{2}$, Takanori Negoro ${ }^{3}$ \\ ${ }^{1}$ Department of Biobased Materials Science, Kyoto Institute of Technology, Kyoto, Japan \\ ${ }^{2}$ Department of Materials and Production Technology Engineering, King Mongkut's University of Technology, North Bangkok, \\ Thailand \\ ${ }^{3}$ NEGORO SANGYO Co., Ltd., Osaka, Japan \\ Email: *vip-wrp@hotmail.com
}

How to cite this paper: Thodsaratpreeyakul, W., Uawongsuwan, P. and Negoro, T. (2018) Properties of Recycled-Polyethylene Terephthalate/Polycarbonate Blend Fabricated by Vented Barrel Injection Molding. Materials Sciences and Applications, 9, 174190.

https://doi.org/10.4236/msa.2018.91012

Received: November 2, 2017

Accepted: January 23, 2018

Published: January 26, 2018

Copyright $\odot 2018$ by authors and Scientific Research Publishing Inc. This work is licensed under the Creative Commons Attribution International License (CC BY 4.0).

http://creativecommons.org/licenses/by/4.0/

\begin{abstract}
In this research, recycled-polyethylene terephthalate (PET) and polycarbonate (RPET/PC) blends fabricated by vented barrel injection molding were presented to better understand the effect of devolatilization during molding process. The effect of dried pellets, non-dried pellets, using an opened-vented hole, and using a closed-vented hole on the miscibility, morphology, thermal properties and mechanical properties of RPET/PC blends was investigated. The results indicated that no drying decreases dispersion, thermal properties, and mechanical properties of RPET/PC blends due to hydrolysis degradation of recycled-PET during the injection molding process. Using the venting system with non-dried RPET/PC blends partially improves dispersion, thermal properties and molecular weight of RPET/PC blends processed without drying, giving results that are similar to those processed with drying. Regarding the flexural properties, using the venting system without drying prevents the flexural properties from decreasing in RPET/PC blends, if the amount of RPET is less than $75 \mathrm{wt} \%$. When the content of RPET is over $75 \mathrm{wt} \%$, using the venting system does not eliminate the decrease in flexural properties of RPET/PC blends. When the venting system is applied to non-dried RPET, despite hydrolysis degradation of RPET not being completely eliminated, the damaging effects are nonetheless reduced compared with those samples processed without the venting system. As a result, vented barrel injection molding hardly prevents non-dried RPET/PC blends from having reduced flexural properties when the content of RPET is greater than $75 \mathrm{wt} \%$.
\end{abstract}




\section{Keywords}

Polymer Blends, Recycled Poly(Ethylene Terephthalate), Polycarbonate, Vented Barrel İnjection Molding

\section{Introduction}

The most important commercial polyester polymer is polyethylene terephthalate (PET), which is widely used because of its high mechanical properties, high transparency, and low cost [1] [2]. The excessively large consumption and disposal of PET-based products, especially in the packaging industry demands that alternatives to successfully recycle and reuse PET products be implemented. Recently, environmental policies for economizing raw materials are promoting the recycling of plastics [3] [4], and great interest is being drawn to recycled-PET (RPET). An interesting method for improving the mechanical and thermal properties of polymer materials is the blend technique [5] [6] [7] [8] [9]. Mechanical recycling by blending PET/RPET with other polymers has shown economic benefit and is a feasible solution for producing new polymer products. The advantages of blending PET and/or RPET with commodity plastics, engineering polymers, and elastomers have now been a number of reported [5] [6] [7] [8]. Depending on the miscibility of the blended polymers, good mechanical and thermal properties can be obtained by mixing, such as high impact strength and high heat deflection temperature (HDT) in the case when combined with polycarbonate (PC) [9].

Srithep et al. [10] studied the effects of chain extenders of a styrene acrylic copolymer having epoxy functional groups on the mechanical and thermal properties of RPET and PC blends. The agents that had a dual function as both a chain extender and a compatibility enhancer, resulted in the improvement of compatibility between RPET and PC and consequently improved mechanical properties of polymer blend. Chen et al. [11] revealed the effect of ethylene glycidyl methacrylate (E-GMA) on the mechanical and morphological properties of recycled HDPE/PET blends. They found that recycled HDPE/PET blends without E-GMA have a larger particle size and poor distribution of dispersed phases with higher contents of PET in the blend. E-GMA increased the interactions between the two phases progressively and enhanced the phase dispersion of the blends. Lin et al. [12] discovered the droplet morphology in RPET/PA6 blends. The incorporation of POE-g-GMA into RPET/PA6 blends subsequently decreased the size of PA6 droplets, and the size of dispersed phase continued to decrease with increasing content of POE-g-GMA. Garcia et al. have reported an increase in transesterification reaction of PET/PC blends by changing processing methods and conditions. The compatibilizing effect via the transesterification reaction can be improved with the using of optimum screw speed, which resulted in an increase in mechanical properties due to improved interchange reactions 
[13]. Transesterification reactions affect the phase structure and mechanical properties of the blends.

As both PET and PC are hygroscopic and they are sensitive to any moisture content that is present. Thus, these materials need to be thoroughly dried before processing, and expensive drying hoppers are required to eliminate any moisture which might be occurred during the process [14]. Because PET and PC readily absorb moisture [15] even they are in atmosphere, removal of moisture during processing by vented barrel injection molding which is likely more effective. The vented barrel injection molding has a vented channel at the middle of the injection barrel that allows volatiles and moisture to be evacuated during processing. Therefore, vented injection molding is suitable for hygroscopic materials as additional drying processes are eliminated, and process ability and mechanical properties of hygroscopic polymer products are improved [16]. In a previous study [17], RPET/PC blends produced by using the vented barrel injection molding revealed the presence of partial immiscibility, which enhances the mechanical properties. Interestingly, the vented barrel injection molding has shown to be effective in producing RPET/PC blends, therefore, the effects of the vented barrel injection molding should be further studied.

In this research, the effect of moisture content and using the vented hole of the vented barrel injection molding on the mixing efficiency of RPET/PC blends was investigated, and RPET, PC and RPET/PC blends were fabricated by the vented barrel injection molding. The effect of dried pellets, non-dried pellets, using an opened-vented hole, and using a closed-vented hole on the miscibility, morphology, thermal properties and mechanical properties of RPET/PC blends was investigated. The advantages of vented barrel injection molding on the mixing efficiency of RPET/PC blends under varying processing conditions are presented.

\section{Experimental}

\subsection{Materials and Specimen Preparation}

Recycled poly(ethylene terephthalate) (RPET) with intrinsic viscosity of $0.65 \mathrm{dl} / \mathrm{g}$ and molecular weight of 12,600 g/mol was supplied by Negoro Sangyo Co., Ltd., Japan. Polycarbonate (PC) with melt flow index of $15 \mathrm{~g} / 10 \mathrm{~min}$ (Iupilon ${ }^{\circledR} \mathrm{S}-3000$ ) was supplied by Mitsubishi Engineering-Plastics Corporation, Japan. The weight ratios of RPET: PC blends were varied at 100:0, 75:25, 50:50, 25:75 and 0:100. The dumbbell samples were dry-blended and fabricated by the vented barrel injection molding machine (TI-30F6, TOYO MACHINERY \& METAL CO., Ltd.). A schematic of the vented barrel injection molding machine is shown in Figure 1.

The specimens were fabricated under four different operating conditions: dried pellet with opened-vented hole (D-V), dried pellet with closed-vented hole (D-NV), non-dried pellet with opened-vented hole open (ND-V) and non-dried pellet with closed-vented hole (ND-NV). The moisture content of RPET and PC 


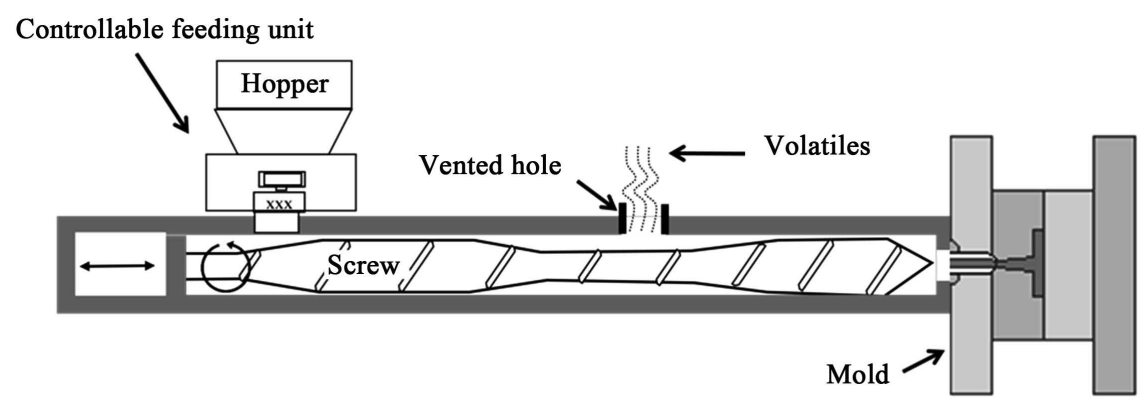

Figure 1. Schematic of the vented barrel injection molding.

pellets before and after drying in an oven was evaluated by a Karl Fisher testing machine. The moisture content of the non-dried RPET and PC was $0.26 \%$ and $0.084 \%$, respectively. After drying at $115^{\circ} \mathrm{C}$ for $12 \mathrm{~h}$, the moisture content of RPET and PC was $0.008 \%$ and $0.005 \%$, respectively.

\subsection{Characterizations}

The observation of phase morphology was performed by a scanning electron microscope (SEM, JEOL/JSM-5200), which was set at $15 \mathrm{kV}$. Gold was sputtered onto the specimens for electron conductivity. The size of the dispersed phase was measured by using the image-J program with the SEM photographs. About 400 particles were measured to determine the diameter of the PC droplets. The average surface diameters were calculated as follows with Equation (1) [18];

$$
\overline{D_{z}}=\sqrt{\frac{\sum N_{i} D_{i}^{2}}{\sum N_{i}}}
$$

The dynamic mechanical properties were performed on a DMA2980 (TA Instruments, USA) using single frequency strain and dual cantilever modes. The DMA was operated under atmospheric conditions at a constant frequency of 1 $\mathrm{Hz}$. The specimens were heated from ambient to $180^{\circ} \mathrm{C}$ with a heating rate of $3^{\circ} \mathrm{C} / \mathrm{min}$.

Differential scanning calorimetry (DSC2920, TA Instruments, USA) was used to characterize thermal properties and crystallinity. Specimens weighing $5 \mathrm{mg}$ were cut from the dumbbells and placed in an aluminum pan. The temperature was set from $30^{\circ} \mathrm{C}-300^{\circ} \mathrm{C}$ with a heating rate of $10^{\circ} \mathrm{C} / \mathrm{min}$ under nitrogen atmosphere. The crystallinity of the RPET polymer was calculated from the following Equation (2);

$$
X_{c}(\%)=\frac{\left(\Delta H_{m}-\Delta H_{c c}\right) \times 100}{\Delta H_{f 100}} \times \frac{1}{W_{p}}
$$

where $X_{c}$ is degree of crystallinity (\%); $\Delta H_{m}$ is enthalpy of melting enthalpy; $\Delta H_{c c}$ is enthalpy of clod crystallization enthalpy; $\Delta H_{\text {fl00 }}$ is the heat of fusion of $100 \%$ PET crystallization (140 J/g [19]) and $W_{p}$ is the weight fraction of the RPET polymer.

For flexural testing, the specimens were cut to dimensions of $60 \times 10 \times 3 \mathrm{~mm}$ 
(length $\times$ width $\times$ thickness) from the dumbbells and the testing speed was 3 $\mathrm{mm} / \mathrm{min}$ with a support span length of $48 \mathrm{~mm}$ according to ASTM D 790 .

Gel permeation chromatography (GPC) were performed, which recorded on a system comprised of a Shimadzu (Kyoto, Japan) LC-20ADVP HPLC pump and a Shimadzu RID-10A differential refractive index detector. Two Showa Denko (Tokyo, Japan) Shodex GPC HFIP-806M columns with a Showa Denko Shodex GPC HFIP-LG guard column were installed in the system, and hexamethylene isopropanol (HFIP) was used as the eluent. The measurements were carried out at $40^{\circ} \mathrm{C}$ at a flow rate of $0.6 \mathrm{ml} \cdot \mathrm{min}^{-1}$. Poly(methyl methacrylate) (PMMA) standards were used to calibrate the molecular weight ranging from 1,500,000 to $1310 \mathrm{Da}$. PC was conducted by a system comprised of Shimadzu GL science with SHIMADZU GPC-802C column. The measurement was done at a flow rate of 1 $\mathrm{ml} \cdot \mathrm{min}^{-1}$. Polystyrene (PS) standards were carried out to calibrate the molecular weight ranging from 650,000 to $2200 \mathrm{Da}$.

\section{Results and Discussion}

\subsection{Phase Morphology Studies of RPET/PC Blends}

The morphology of RPET/PC blends fabricated by the vented barrel injection molding was investigated by scanning electron microscopy (SEM). SEM photographs for all RPET/PC blends under the four different conditions are presented in Figure 2. In the RPET/PC blends without a catalyst, the SEM photographs show a binary structure at all blending compositions. The morphologies of the different compositions of PC in RPET/PC blends under D-NV are shown in Figure 2(a-3), Figure 2(b-3) and Figure 2(c-3). Droplet morphology of minor phase is observed in the composition range of $25 \%$ PC, 50\% PC and 75\% PC.

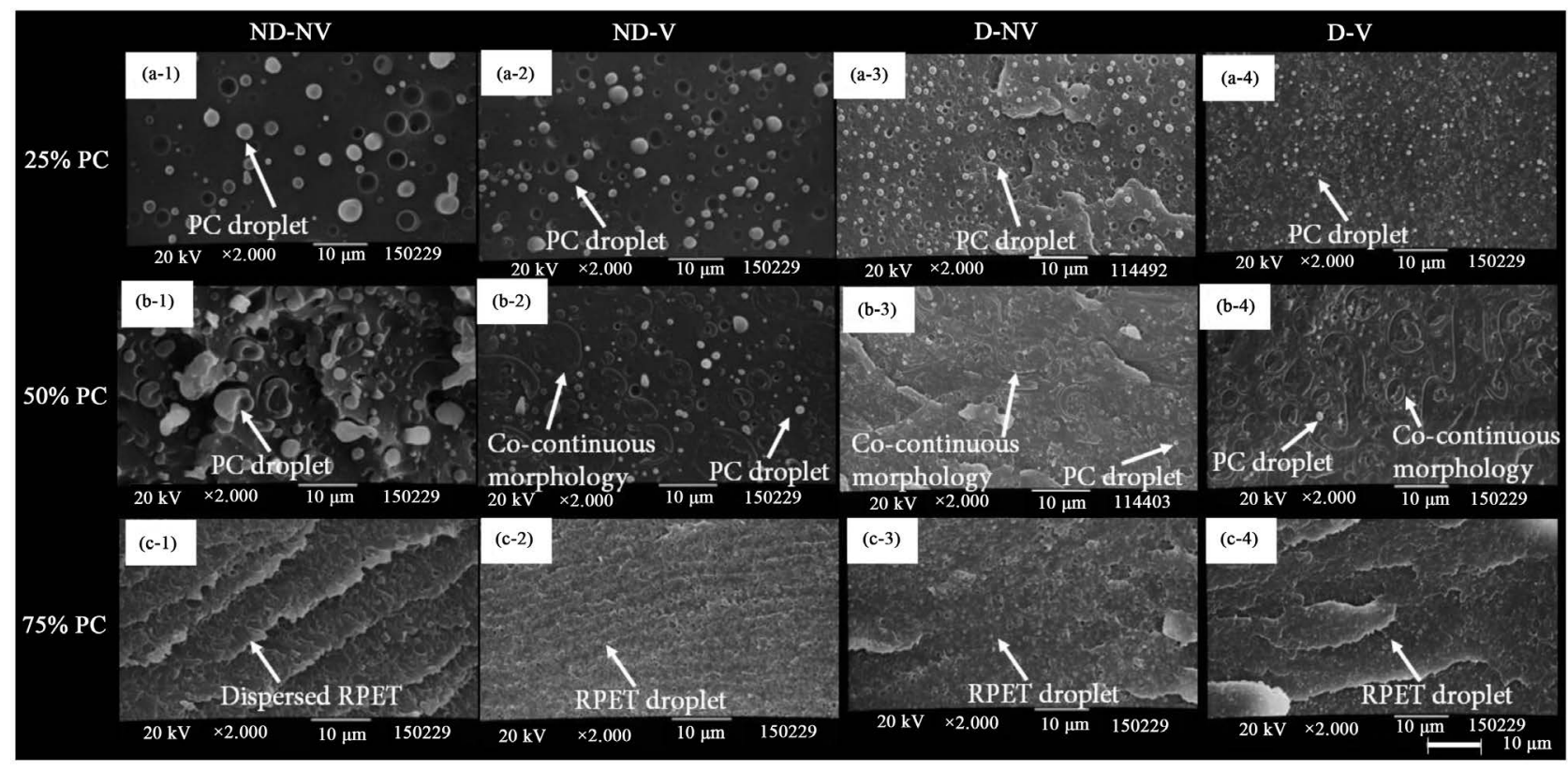

Figure 2. SEM photographs of RPET/PC blends under the four different conditions: $25 \%$ PC, $50 \%$ PC and $75 \%$ PC were coded by (a), (b) and (c), respectively; ND-NV, ND-V, D-NV and D-V were coded by 1, 2, 3 and 4 respectively. 
Many researchers revealed that the droplet morphology is generally found in a binary structure at a larger composition range, such as in COC/POE blends and PP/PC blends as revealed by Khonakdar et al. [20] and Laoutid et al. [21], respectively. A co-continuous morphology with small droplets can be found only in 50\% PC as shown in Figure 2(b). Mbarek et al. have also reported that a cocontinuous morphology is only found in a 50/50 blend composition of PET/PC [22].

When comparing the SEM photographs of RPET/PC blends under each conditions, it is evident that non-dried RPET/PC blends with closed-vented hole (ND-NV) is the worst distribution of RPET or PC minor phase. Figure 2(a-3), Figure 2(b-3) and Figure 2(c-3) indicate that drying strongly influences the mixing behavior of RPET/PC blends, affecting the compatibilizing effect of interchange reactions. The dispersed PC droplets in 25\% PC ND-NV is larger than those of D-NV. For ND-NV at 50\% PC, the co-continuous morphology is decreased and the dispersed PC droplets are larger when compared with D-NV, Figure 2(b). The dispersed RPET phase in 75\% PC ND-NV is large with no observable pattern; in contrast to 75\% PC D-NV, where the dispersed RPET phase consists of, forming the droplet morphology shown in Figure 2(c). The influence of the vented hole of the vented barrel injection molding on the morphology of RPET/PC blends were investigated by comparing between the blends made with open and close vented hole. The opened-vented hole of the vented barrel injection molding improves the mixing of RPET/PC blends when compared with those made with closed-vented hole. The dispersion of RPET/PC blends has an improved droplet morphology, consisting of a co-continuous morphology dispersion when the vented hole is open as similar to those with drying, Figure 2(a-2), Figure 2(b-2) and Figure 2(c-2). Using opened-vented hole with dried RPET/PC blends (D-V) slightly gives the better distribution of minor phase when compared with those of dried RPET/PC blends with closed-vented hole (D-NV) as shown in Figure 2(a-4), Figure 2(b-4) and Figure 2(c-4).

To verify the effect of drying and opening the vented hole, the size of the dispersed PC droplets in 25\% PC were measured by Image-J software, and then the average surface diameter of $25 \%$ PC at various processing conditions was calculated. The cumulative percentage of droplets sizes and the calculated average surface diameter are shown in Figure 3. Non-dried RPET/PC lead to bigger size of dispersed PC droplets. Drying can decrease the dispersed PC droplet size in RPET/PC blends. Opening the vented hole is also significantly effective in the dispersion of RPET/PC blends. The PC droplets size of ND-V has becomes close to D-NV, and the PC droplets size of D-V is slightly less than D-NV. Garcia et al. [13] revealed the compatibilizing effect of interchange reactions of PET/PC blends without a catalyst demonstrated by the size of dispersed particles. The authors disclosed that the smaller the dispersed droplets are, the better the compatibilizing effect of interchange reactions of PET/PC blends without a catalyst. 


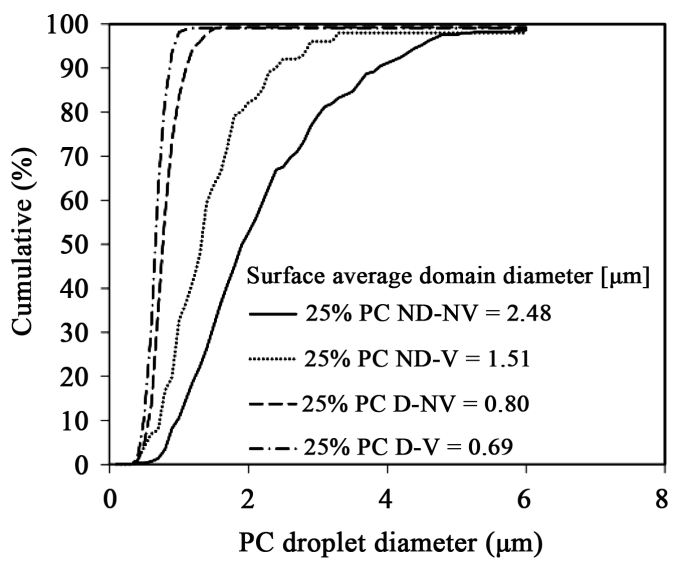

Figure 3. The diameter cumulative and average surface domain diameter of $25 \%$ PC under four different conditions.

\subsection{Dynamic Mechanical Analysis and Standard Differential Scanning Calorimetry Measurements}

The effects of drying and opening the vented hole on dynamic mechanical properties of RPET/PC blends were verified by Dynamic Mechanical Analysis (DMA). DMA is the useful technique, which the effect of dispersion and interfacial interaction can be effectively detected, especially by observation at the measured storage modulus [23]. Figure 4 and Figure 5 show the storage modulus and tan delta plots that were measured for neat RPET and PC and for 25\% PC, 50\% PC and 75\% PC under different processing conditions. As can be seen in Figure 4, the storage modulus show three distinct regions referring as the glassy high modulus region, the transition zone and the rubbery low modulus region. RPET has higher storage modulus when comparing with neat PC. The storage modulus of blends is higher than both neat RPET and PC at all composition. The blend show transition and plateau region corresponding to RPET and PC phase. At all compositions of blends, ND-NV has the lowest storage modulus when compared to the others that were dried; however, opening the vented hole (ND-V) increases the storage modulus of non-dried RPET/PC blends as well as those that are dried. In case of dried materials (D-NV and D-V), opening the vented hole does not show any effect on the storage modulus of RPET/PC blends.

Tan delta plots are shown in Figure 5, and two main peaks are observed in the RPET/PC blends, with the first peak corresponding to the phase transition of RPET at its $T_{g}$, and the second peak corresponding to the $T_{g}$ of PC. And between the peaks of RPET and PC for all blends, smaller tan delta peaks were also observed at a temperature of $120^{\circ} \mathrm{C}$, which corresponds to the crystallization temperature of PET. The DMA results indicate that RPET/PC blends are immiscible, as represented by the two tan delta peaks.

The lower intensity of the tan delta peaks indicates better dispersion of the dispersed phase in RPET/PC blends [24] [25] [26], because there are more restrict the molecular mobility. And the smaller particles in the dispersed phase would be better to enhance the modulus of the blends [27] [28] [29], The intensity of the tan 


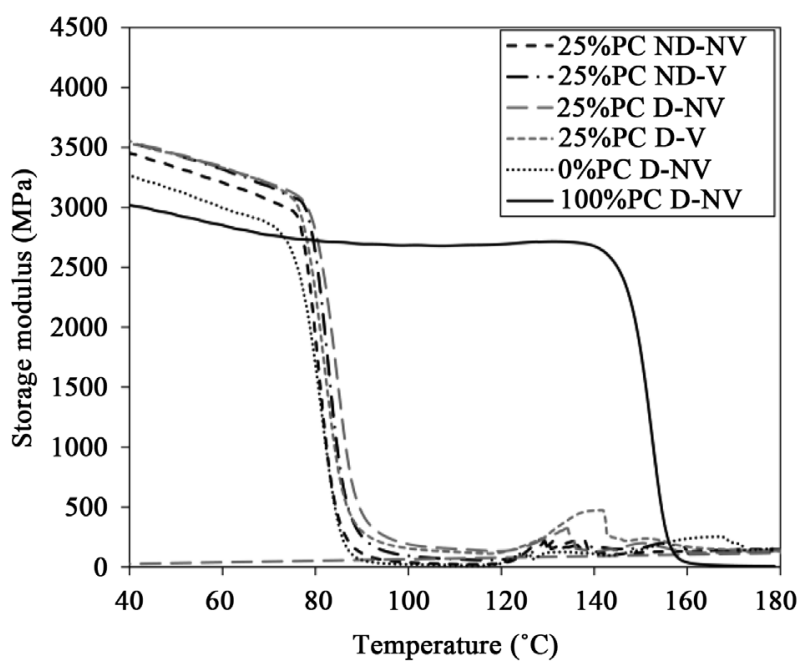

(a)

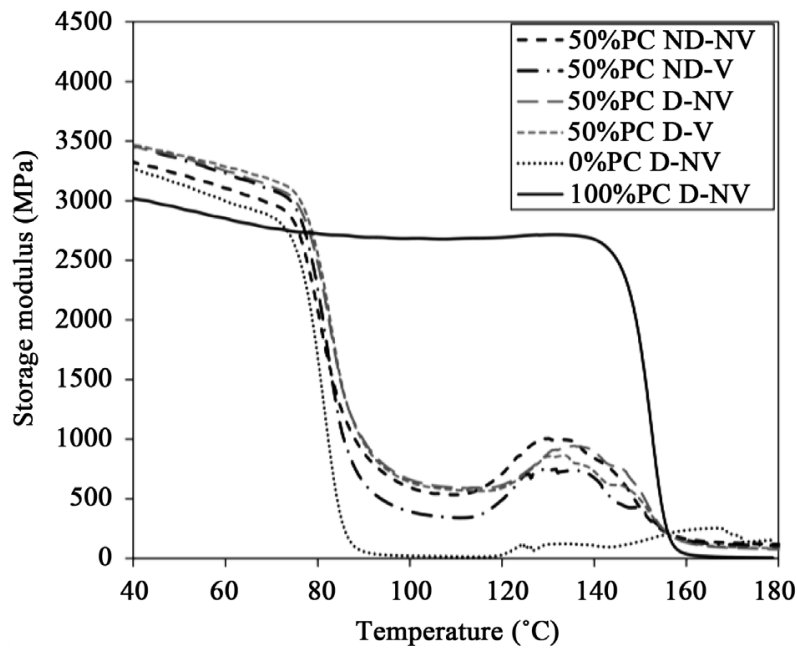

(b)

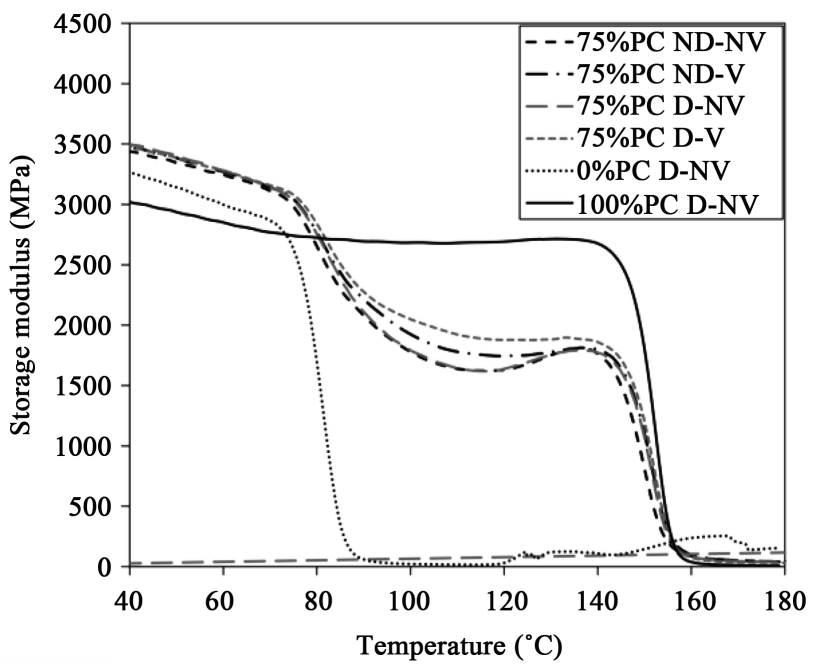

(c)

Figure 4. Storage modulus of (a) 25\% PC; (b) 50\% PC and (c) 75\% PC under four different conditions. 


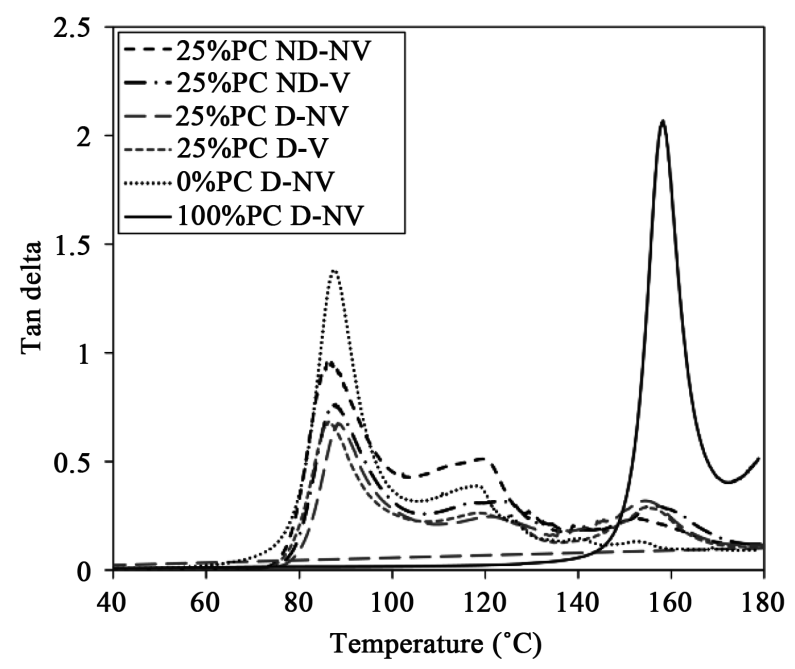

(a)

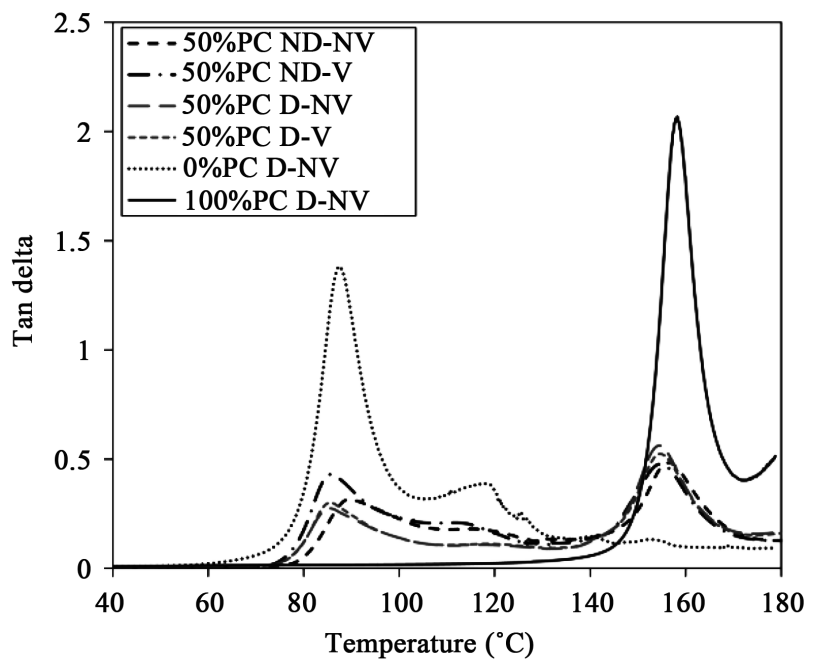

(b)

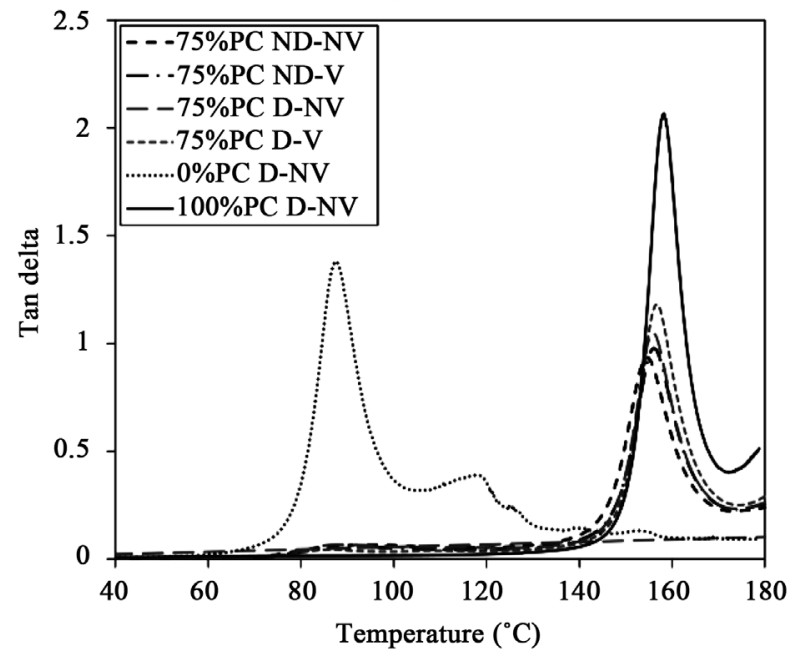

(c)

Figure 5. Tan delta of (a) 25\% PC; (b) 50\% PC and (c) 75\% PC under four different conditions. 
delta peaks of the RPET/PC blends is noticeably decreased compared to those of neat RPET and PC, which indicates the existence of good dispersion in the blended RPET, or that PC restrained the molecular mobility of the polymer matrix during transition. In Figure 5, the intensity of the tan delta curves of RPET/ PC blends under each conditions shows that where non-dried samples result in higher intensity of the tan delta peak heights compared to the dried samples, and where opening the vented hole decreases the intensity of the tan delta peak heights, being closer to the results of the dried condition. Figure 6 and Figure 7 show DSC plots of the different processing conditions for RPET, PC and for RPET/PC blends and Table 1 shows $\mathrm{T}_{\mathrm{g}}, \mathrm{T}_{\mathrm{cc}}$ and $\mathrm{T}_{\mathrm{m}}$ values determined by DSC. The value of RPET's $\mathrm{T}_{\mathrm{g}}$ for D-NV RPET was $74^{\circ} \mathrm{C}$, and for D-NV 25\% PC, 50\% PC and $75 \%$ PC, the RPET's $\mathrm{T}_{\mathrm{g}}$ values have increased to $75.6^{\circ} \mathrm{C}, 77.8^{\circ} \mathrm{C}$ and $78.1^{\circ} \mathrm{C}$, respectively. The $\mathrm{T}_{\mathrm{g}}$ value of $\mathrm{PC}$ in $\mathrm{D}-\mathrm{NV}$ PC was $150.6^{\circ} \mathrm{C}$, and the values

Table 1. The calculated crystallinity, glass transition, cold crystallization temperature and melting temperature of PC/RPET blends.

\begin{tabular}{|c|c|c|c|c|c|c|}
\hline & & $\mathrm{X}_{c}(\%)$ & $\mathrm{T}_{\mathrm{g}} \operatorname{RPET}\left({ }^{\circ} \mathrm{C}\right)$ & $\mathrm{T}_{\mathrm{g}} \mathrm{PC}\left({ }^{\circ} \mathrm{C}\right)$ & $\mathrm{T}_{\mathrm{cc}}\left({ }^{\circ} \mathrm{C}\right)$ & $\mathrm{T}_{\mathrm{m}}\left({ }^{\circ} \mathrm{C}\right)$ \\
\hline \multirow{4}{*}{$0 \% \mathrm{PC}$} & ND-NV & 13.9 & 71.2 & - & 122.2 & 255.3 \\
\hline & $\mathrm{ND}-\mathrm{V}$ & 13.9 & 73.5 & - & 125.1 & 254.4 \\
\hline & D-NV & 12.2 & 74.2 & - & 126.8 & 252.6 \\
\hline & $\mathrm{D}-\mathrm{V}$ & 12.0 & 74.5 & - & 127.3 & 253.3 \\
\hline \multirow{4}{*}{$25 \% \mathrm{PC}$} & ND-NV & 14.9 & 72.5 & Un & 125.4 & 254.5 \\
\hline & $\mathrm{ND}-\mathrm{V}$ & 14.3 & 73.3 & Un & 124.0 & 253.4 \\
\hline & D-NV & 11.9 & 75.6 & Un & 132.7 & 251.7 \\
\hline & $\mathrm{D}-\mathrm{V}$ & 13.8 & 75.4 & Un & 130.9 & 251.2 \\
\hline \multirow{4}{*}{$50 \% \mathrm{PC}$} & ND-NV & 16.8 & 72.7 & 145.4 & 123.2 & 254.2 \\
\hline & ND-V & 13.7 & 74.1 & 146.9 & 126.8 & 253.7 \\
\hline & $\mathrm{D}-\mathrm{NV}$ & 9.8 & 77.8 & Un & 136.6 & 249.1 \\
\hline & $\mathrm{D}-\mathrm{V}$ & 9.4 & 76.8 & Un & 137.4 & 251.3 \\
\hline \multirow{4}{*}{$75 \%$ PC } & ND-NV & 13.9 & 74.8 & 146.5 & 124.6 & 254.2 \\
\hline & ND-V & 13.2 & 78.3 & 147.8 & 124.9 & 253.7 \\
\hline & D-NV & 11.8 & 78.1 & 148.3 & 134.6 & 250.7 \\
\hline & $\mathrm{D}-\mathrm{V}$ & 8.0 & 78.1 & 148.4 & 134.4 & 251.0 \\
\hline \multirow{4}{*}{$100 \%$ PC } & ND-NV & - & - & 150.6 & - & - \\
\hline & ND-V & - & - & 151.0 & - & - \\
\hline & D-NV & - & - & 150.6 & - & - \\
\hline & $\mathrm{D}-\mathrm{V}$ & - & - & 150.8 & - & - \\
\hline
\end{tabular}

Un: Untraceable. 


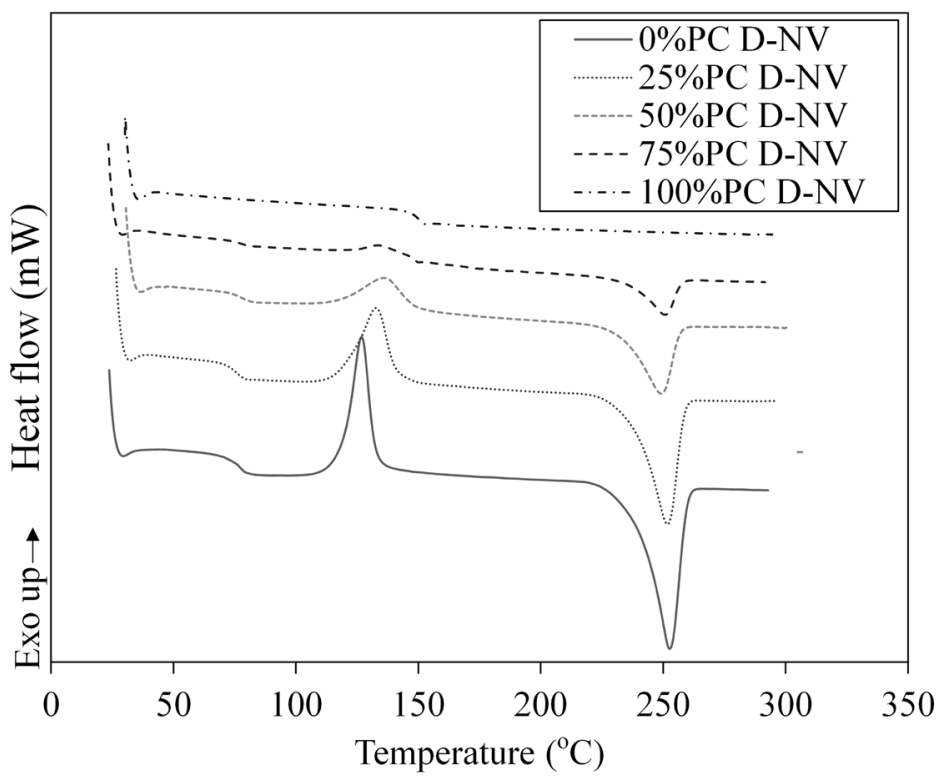

Figure 6. DSC thermograms of RPET/PC blends at various PC ratios under D-NV condition.

of $\mathrm{T}_{\mathrm{g}}$ for PC significantly decrease in the RPET/PC blends. The values of RPET's $\mathrm{T}_{\mathrm{cc}}$ for RPET and the blends show a similarly trend to the values of $\mathrm{T}_{\mathrm{g}} \cdot \mathrm{T}_{\mathrm{g}}$ and $\mathrm{T}_{\mathrm{cc}}$ values of RPET and RPET/PC blends decrease when they are processed with no drying; however, the decrease in $\mathrm{T}_{\mathrm{g}}$ and $\mathrm{T}_{\mathrm{cc}}$ values of those made with no drying can be reduced by opening the vented hole. RPET and RPET/PC blends with no-drying, gives a higher degree of crystallinity of RPET when compared to those with drying, as shown in Figure 7.

\subsection{Mechanical Properties}

From the flexural tests, load-displacement curves are shown in Figure 8 and the flexural modulus and strength are presented in Figure 9. Using ND-NV, it affects slightly the flexural properties of PC, but it significantly influences the flexural properties of RPET. Even the use of ND-NV with RPET increases the flexural modulus and strength of RPET, however the fracture behavior of RPET ND-NV becomes brittle and fragile. Opening the vented hole with non-dried RPET (ND-V) gives similar flexural properties to those of D-NV, and also provides ductile fracture that is observed in dried RPET. Interestingly, using the open vented hole with dried RPET or PC increases flexural properties of RPET and PC when compared with those of D-NV. In the case of RPET/PC blends, using ND-NV leads to a decrease in flexural properties unless PC is incorporated at more than $75 \mathrm{wt} \%$. The flexural properties of non-died RPET/PC blends, which contains less than $75 \mathrm{wt} \%$ PC, can be improved by opening the vented hole so that they are similar to those of D-NV. However, using the open vented hole with non-dried RPET/PC blends (ND-V) is inefficient when the incorporated PC is less than $25 \mathrm{wt} \%$. 


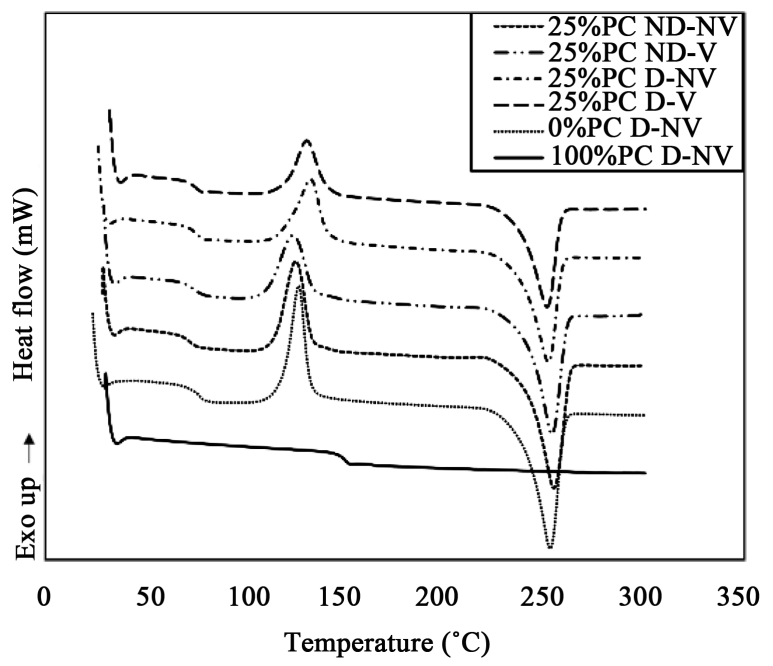

(a)

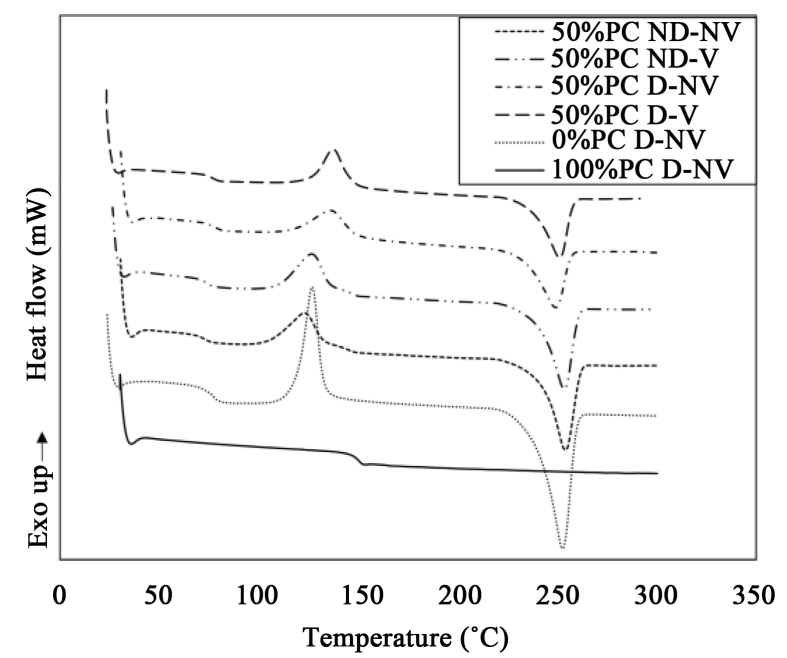

(b)

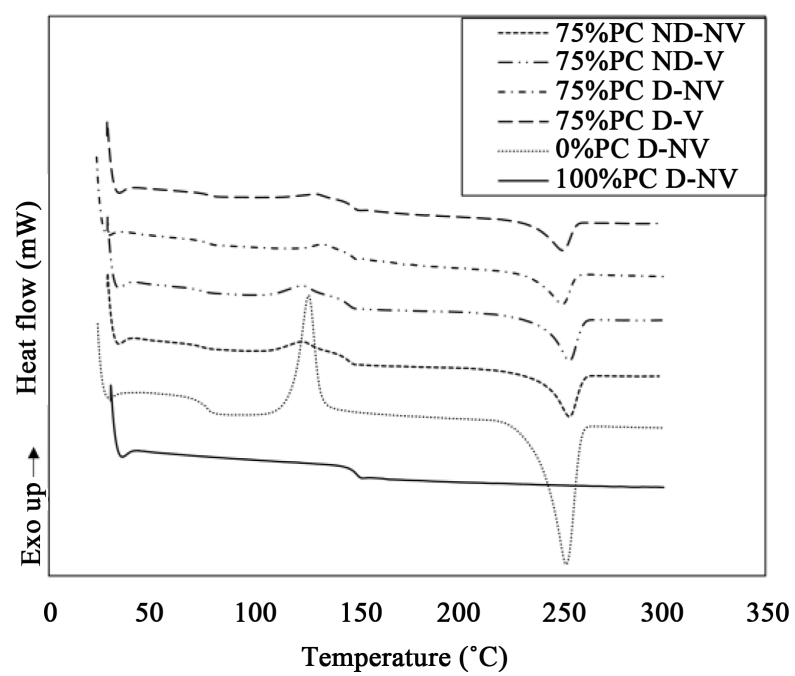

(c)

Figure 7. DSC thermograms of (a) 25\% PC; (b) 50\% PC and (c) $75 \%$ PC under four different conditions. 


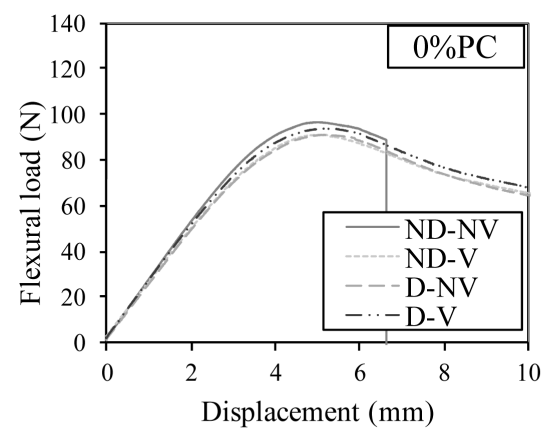

(a)

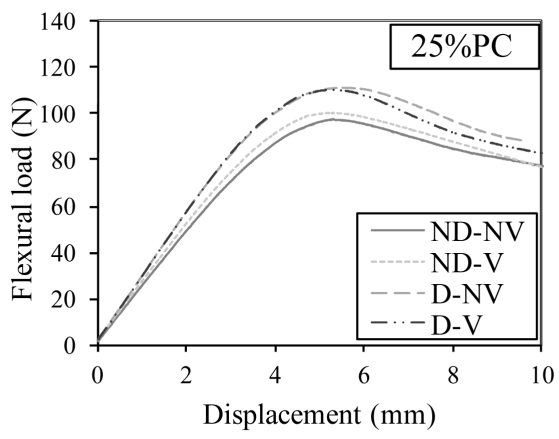

(b)

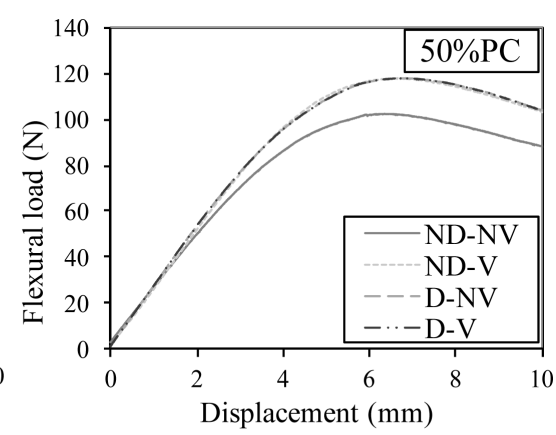

(c)

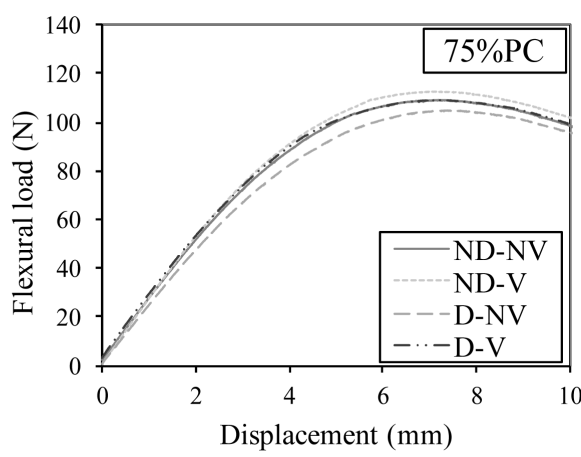

(d)

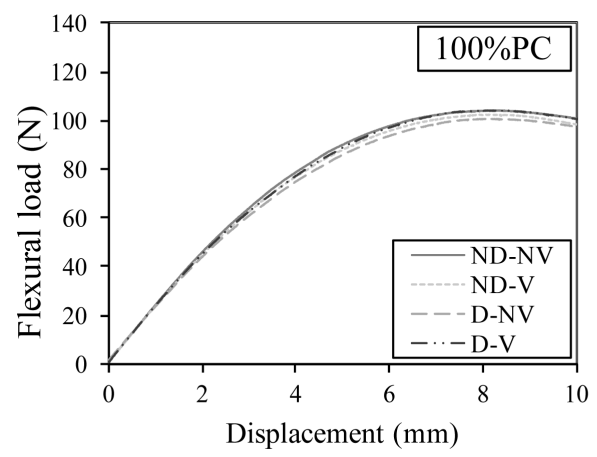

(e)

Figure 8. Load-displacement curves of (a) RPET; (b) 25\% PC; (c) 50\% PC; (d) 75\% PC and (E) 100\% PC under four different conditions.

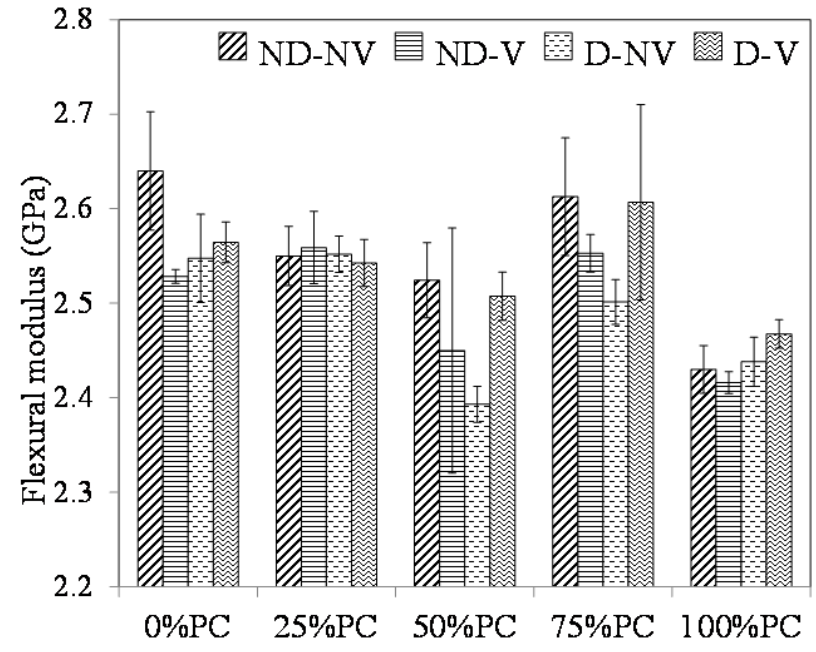

(a)

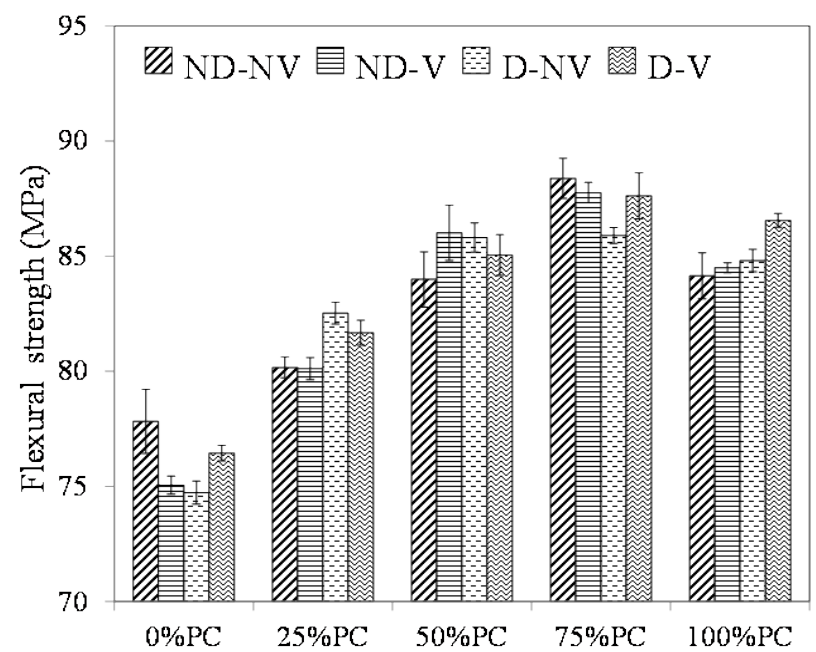

(b)

Figure 9. Flexural modulus (a) and flexural strength; (b) of RPET, PC and the RPET/PC blends under four different processing conditions.

\subsection{Molecular Weight Determination}

The number average molecular weight $\left(M_{n}\right)$, the weight average molecular weight $\left(M_{w}\right)$, and the polydispersion index (PDI) of RPET and PC in both as-received and injection-molded samples at various conditions obtained by GPC are provided in Table 2. It is observed that drying preserves the as-received 
Table 2. The number- and the weight-average molecular weights and the polydispersion index, $M_{\mathrm{n}}, M_{\mathrm{w}}$, and PDI of RPET.

\begin{tabular}{cccc}
\hline Samples & $M_{\mathrm{n}}$ & $M_{\mathrm{w}}$ & PDI \\
\hline As-received RPET & 7650 & 12,500 & 1.63 \\
RPET ND-NV & 4180 & 6680 & 1.60 \\
RPET ND-V & 5540 & 8680 & 1.57 \\
RPET D-NV & 7420 & 12,100 & 1.63 \\
RPET D-V & 7320 & 12,000 & 1.63 \\
As-received PC & 21,500 & 50,000 & 2.34 \\
PC ND-NV & 21,200 & 46,300 & 2.18 \\
PC ND-V & 22,000 & 48,600 & 2.13 \\
PC D-NV & 22,800 & 48,400 & 2.18 \\
PC D-V & 22,800 & 49,700 & 2.14 \\
\hline
\end{tabular}

molecular weight of RPET in both D-NV and D-V RPET. On the other hand, performing injection molding with no drying in ND-NV decreases the $M_{w}$ of RPET up to $47 \%$ as compared to the as-received RPET. When the vented hole is open, $M_{w}$ of RPET ND-V decreases less than that of RPET ND-NV. The molecular weight of ND-V RPET decreased by $30 \%$ compared to the as-received RPET. On the other hand, in the case of PC, the molecular weight in ND-NV is reduced by $7 \%$ compared to the as-received PC. Opening the vented hole with non-dried PC (ND-V) yields a $M_{w}$ that is very similar to that of the as-received PC. From the results, it is clear that hydrolysis degradation has less of an effect on PC compared to RPET. The open vented hole exhibits sufficient performance to prevent the decrease in molecular weight of non-dried PC (ND-V). As a result, when the PC content of RPET/PC is high, using the vented hole can prevent any decrease in mechanical properties from hydrolysis degradation. However, the devolatilization efficiency of vented barrel injection molding has to be further developed to better prevent hydrolysis degradation of RPET during processing of RPET/PC blends.

The GPC results confirm the reason for the increase in crystallinity of nondried RPET and the blends, which have a higher flexural modulus than those of D-NV. Non-drying leads to hydrolysis degradation, which decreases the molecular weight of RPET and RPET/PC blends and facilitates molecular chain arrangement. Moreover, previous research [30] [31] [32] revealed that the presence of moisture in RPET/PC blends that are not dried during processing influences the kinetics of crystallization of RPET, since moisture decreases an introduction time of crystallization and half-time of crystallization caused the easy crystallization. Even if injection molding is performed without drying and crystallinity increases of the RPET and blends increases, hydrolysis degradation of RPET significantly decreases the mechanical properties. 


\section{Conclusion}

No drying decreases dispersion, thermal properties, and mechanical properties of RPET/PC blends due to hydrolysis degradation during the injection molding process. Using the venting system with non-dried materials partially improves dispersion, thermal properties and molecular weight of RPET/PC blends processed without drying, giving results that are similar to those processed with drying. Regarding the flexural properties, using the venting system without drying prevents the flexural properties from decreasing in RPET/PC blends, if the amount of RPET is less than $75 \mathrm{wt} \%$. When the content of RPET is over $75 \mathrm{wt} \%$, using the venting system does not eliminate the decrease in mechanical properties of RPET/PC blends. The open vented hole exhibits sufficient performance to prevent the decrease in molecular weight of non-dried PC. As a result, when the $\mathrm{PC}$ content of RPET/PC is high, using the vented hole can prevent any decrease in mechanical properties from hydrolysis degradation. When the venting system is applied to non-dried RPET, despite hydrolysis degradation of RPET not being completely eliminated, the damaging effects are nonetheless reduced compared with those samples processed without the venting system. As a result, vented barrel injection molding hardly prevents non-dried RPET/PC blends from having reduced flexural properties when the content of RPET is greater than 75 $w t \%$. Thus, the operating conditions for vented barrel injection molding must be further studied to prevent hydrolysis degradation, as it concerns the processing of RPET/PC blends. The effect of barrel temperature at the positions before the vented hole of the vented barrel injection molding should be investigated in future studies for improving devolatilization.

\section{References}

[1] Albertsson, A.C. and Karlsson, S. (1993) Degradable Polymers. Pure and Applied Chemistry, 30, 757-765. http://doi.org/10.1080/10601329308021261

[2] Awaja, F. and Pavel, D. (2005) Recycling of PET. European Polymer Journal, 41, 1453-1477. https://doi.org/10.1016/j.eurpolymj.2005.02.005

[3] Nassar, T.R., Paul, D.R. and Barlow, J.W. (1979) Polyester-Polycarbonate Blends. II. Poly(Ethylene Terephthalate). Journal of Applied Polymer Science, 23, 85-99. https://doi.org/10.1002/app.1979.070230108

[4] Barlow, J.W. and Paul, D.R. (1981) Polymer Blends and Alloys-A Review of Selected Considerations. Polymer Engineering \& Science, 21, 985-996.

https://doi.org/10.1002/pen.760211502

[5] Srithep, Y., Javadi, A., Pilla, S., Turng, L.S., Gong, S., Clemons, C. and Peng, J. (2011) Processing and Characterization of Recycled Poly(Ethylene Terephthalate) Blends with Chain Extenders, Thermoplastic Elastomer, and/or Poly(Butylene Adipate-co-Terephthalate). Polymer Engineering \& Science, 51, 1023-1032. https://doi.org/10.1002/pen.21916

[6] Entezam, M., Khonakdar, H.A. and Yousefi, A.A. (2013) On the Flame Resistance Behavior of PP/PET Blends in the Presence of Nanoclay and a Halogen Free Flame Retardant. Macromolecular Materials and Engineering, 298, 1074-1084. https://doi.org/10.1002/mame.201200195 
[7] Xie, L., Xie, Y., Wu, Q., Wang, M., Wu, Q., Zhou, X. and Ge, X. (2015) Effect of Poly(Acrylic Acid)-Modified Poly(Ethylene Terephthalate) on Improving the Integrated Mechanical Properties of Poly(Ethylene Terephthalate)/Elastomer Blend. Industrial \& Engineering Chemistry Research, 54, 4748-4755. https://doi.org/10.1021/acs.iecr.5b00091

[8] Imamura, N., Sakamoto, H., Higuchi, Y., Yamamoto, H., Kawasaki, S., Yamada, K., Nishimura, H. and Nishino, T. (2014) Effectiveness of Compatibilizer on Mechanical Properties of Recycled PET Blends with PE, PP, and PS. Materials Sciences and Applications, 5, 548. https://doi.org/10.4236/msa.2014.58057

[9] Lin, L., Deng, C. and Wang, Y.Z. (2015) Improving the Impact Property and Heat Resistance of PLA/PC Blends through Coupling Molecular Chains at the Interface. Polymers for Advanced Technologies, 26, 1247-1258. https://doi.org/10.1002/pat.3560

[10] Srithep, Y., Pholharn, D., Dassakorn, A. and Morris, J. (2017) Effect of Chain Extenders on Mechanical and Thermal Properties of Recycled Poly(Ethylene Terephthalate) and Polycarbonate Blends. Materials Science and Engineering Conference Series, 213, 012008. https://doi.org/10.1088/1757-899X/213/1/012008

[11] Chen, R.S., Ab Ghani, M.H., Salleh, M.N., Ahmad, S. and Gan, S. (2014) Influence of Blend Composition and Compatibilizer on Mechanical and Morphological Properties of Recycled HDPE/PET Blends. Materials Sciences and Applications, 5, 943952. https://doi.org/10.4236/msa.2014.513096

[12] Lin, X., Qian, Q., Xiao, L., Chen, Q., Huang, Q. and Zhang, H. (2014) Influence of Reactive Compatibilizer on the Morphology, Rheological, and Mechanical Properties of Recycled Poly(Ethylene Terephthalate)/Polyamide 6 Blends. Journal of Macro-molecular Science, Part B, 53, 1543-1552. https://doi.org/10.1080/00222348.2014.946840

[13] Garcia, M., Eguiazabal, J.I. and Nazabal, J. (2001) Effects of Mixing Time on Phase Structure and Mechanical Properties of Poly (Ethylene Terephthalate)/Polycarbonate Blends. Journal of Applied Polymer Science, 81, 121-127.

https://doi.org/10.1002/app.1421

[14] Rosato, D.V. and Rosato, M.G. (2012) Injection Molding Handbook. Springer Science \& Business Media, Berlin.

[15] Jabarin, S.A. and Lofgren, E.A. (1986) Effects of Water Absorption on Physical Properties and Degree of Molecular Orientation of Poly(Ethylene Terephthalate). Polymer Engineering \& Science, 26, 620-625. https://doi.org/10.1002/pen.760260907

[16] Hemmati, M. and Nunn, R.E. (1990) Vented Barrel Injection Molding of PC and a PC/ABS Blend. The Free Library. https://www.thefreelibrary.com/VentedbarrelinjectionmoldingofPCandaPC/ABSble nd.-a09316262

[17] Negoro, T., Wongpajan, R., Thodsaratpreeyakul, W., Boonlertsamut, J., Thumsorn, S., Inoya, H. and Hamada, H. (2017) Optimizing of Vented Injection Molding on Mechanical Performance and Miscibility of Recycled Poly(Ethylene Terephthalate) and Polycarbonate Blends. Journal of Polymer Engineering, 37, 271-277. https://doi.org/10.1515/polyeng-2016-0034

[18] Jose, S., Aprem, A.S., Francis, B., Chandy, M.C., Werner, P., Alstaedt, V. and Thomas, S. (2004) Phase Morphology, Crystallisation Behaviour and Mechanical Properties of Isotactic Polypropylene/High Density Polyethylene Blends. European Polymer Journal, 40, 2105-2115. https://doi.org/10.1016/j.eurpolymj.2004.02.026 
[19] Bimestre, B.H. and Saron, C. (2012) Chain Extension of Poly (Ethylene Terephthalate) by Reactive Extrusion with Secondary Stabilizer. Materials Research, 15, 467 472. https://doi.org/10.1590/S1516-14392012005000058

[20] Khonakdar, H.A., Jafari, S.H. and Hesabi, M.N. (2015) Miscibility Analysis, Viscoelastic Properties and Morphology of Cyclic Olefin Copolymer/Polyolefin Elastomer (COC/POE) Blends. Composites Part B: Engineering, 69, 111-119. https://doi.org/10.1016/j.compositesb.2014.09.034

[21] Laoutid, F., Estrada, E., Michell, R.M., Bonnaud, L., Müller, A.J. and Dubois, P. (2013) The Influence of Nanosilica on the Nucleation, Crystallization and Tensile Properties of PP-PC and PP-PA Blends. Polymer, 54, 3982-3993. https://doi.org/10.1016/j.polymer.2013.05.031

[22] Mbarek, S., Jaziri, M. and Carrot, C. (2006) Recycling Poly (Ethylene Terephtalate) Wastes: Properties of Poly(Ethylene Terephtalate)/Polycarbonate Blends and the Effect of a Transesterification Catalyst. Polymer Engineering \& Science, 46, 1378 1386. https://doi.org/10.1002/pen.20625

[23] Zhou, M., Yan, J., Li, Y., Geng, C., He, C., Wang, K. and Fu, Q. (2013) Interfacial Strength and Mechanical Properties of Biocomposites Based on Ramie Fibers and Poly(Butylene Succinate). RSC Advances, 3, 26418-26426.

[24] Hassan, E., Wei, Y., Jiao, H. and Muhuo, Y. (2013) Dynamic Mechanical Properties and Thermal Stability of Poly(Lactic Acid) and Poly(Butylene Succinate) Blends Composites. Journal of Fiber Bioengineering and Informatics, 6, 85-94. https://doi.org/10.3993/jfbi03201308

[25] Poletto, M. (2016) Polystyrene Cellulose Fiber Composites: Effect of the Processing Conditions on Mechanical and Dynamic Mechanical Properties. Matéria (Rio de Janeiro), 21, 552-559. https://doi.org/10.1590/S1517-707620160003.0053

[26] Sankaran, K., Nando, G.B., Ramachandran, P., Nair, S., Govindan, U., Arayambath, S. and Chattopadhyay, S. (2015) Influence of Hybrid Nanostructures and Its Tailoring Mechanism on Permeability, Rheology, Conductivity, and Adhesion Properties of a Novel Rubber Blend Nanocomposite. RSC Advances, 5, 87864-87875. https://doi.org/10.1039/C5RA17178D

[27] Joseph, S., Bambola, V.A., Sherhtukade, V.V. and Mahanwar, P.A. (2011) Effect of Flyash Content, Particle Size of Flyash, and Type of Silane Coupling Agents on the Properties of Recycled Poly(Ethylene Terephthalate)/Flyash Composites. Journal of Applied Polymer Science, 119, 201-208. https://doi.org/10.1002/app.32449

[28] Lin, Z. and Mai, K. (2007) Effect of Inorganic Filler on the Crystallization, Mechanical Properties and Rheological Behavior of Poly(Trimethylene Terephthalate). Polymer-Plastics Technology and Engineering, 46, 417-420. https://doi.org/10.1080/03602550701244600

[29] Thumsorn, S., Yamada, K., Leong, Y.W. and Hamada, H. (2013) Thermal Decomposition Kinetic and Flame Retardancy of $\mathrm{CaCO}_{3}$ Filled Recycled Polyethylene Terephthalate/Recycled Polypropylene Blend. Journal of Applied Polymer Science, 127, 1245-1256. https://doi.org/10.1002/app.37673

[30] Jabarin, S.A. (1987) Crystallization Kinetics of Polyethylene Terephthalate. I. Isothermal Crystallization from the Melt. Journal of Applied Polymer Science, 34, 8596. https://doi.org/10.1002/app.1987.070340107

[31] Jabarin, S.A. (1987) Crystallization Kinetics of Polyethylene Terephthalate. II. Dynamic Crystallization of PET. Journal of Applied Polymer Science, 34, 97-102. https://doi.org/10.1002/app.1987.070340108

[32] Demirel, B., Yaraş, A. and Elçiçek, H. (2016) Crystallization Behavior of PET Materials. Balıkesir Üniversitesi Fen Bilimleri Enstitüsü Dergisi, 13, 26-35. 